\title{
Sleep apnoea and its impact on public health
}

\author{
John Wright, Trevor Sheldon
}

In 1997 we published a systematic review on the health effects of obstructive sleep apnoea and the effectiveness of treatment with CPAP. ${ }^{1}$ This generated much controversy and considerable correspondence. About half the letters received by the authors suggested that the paper was just stating the obvious, the other half that the paper was completely inaccurate. To help clarify our findings this paper will discuss the public health impact of sleep apnoea and the rationale behind systematic reviews.

In recent years several traditional style reviews in leading medical journals have suggested the importance of sleep apnoea. ${ }^{2-4}$ These have claimed that sleep apnoea causes premature death, hypertension, heart disease, stroke, pulmonary hypertension, and road traffic accidents. The high prevalence of $2-4 \%$ of the middle aged population ${ }^{5}$ led an editorial writer in the New England Fournal of Medicine to claim that the "staggering" impact of sleep apnoea was as big a public health hazard as smoking. ${ }^{2}$ If so, sleep apnoea would clearly be of major public concern.

The problem with traditional reviews is that they can be haphazard, biased and reflect the personal prior beliefs or interests of individual reviewers. ${ }^{6}$ Systematic reviews apply strict scientific criteria in an attempt to reduce this bias. They provide a more objective approach to summarising the large number of biomedical research papers and allow us to identify gaps in the research. In addition, systematic reviews can help increase understanding about the generalisability, consistency, and precision of results of individual trials. This is not the same as "evidence based medicine" but they can inform individual clinical decisions as well as health policy.

Like all good science, systematic reviews are based on rigorous methodology. This includes: (1) construction of a clearly focused question; (2) explicit and thorough search of the literature including non-English language papers and the "grey" literature (conference abstracts, reports), as well as contact with known experts in the field to identify relevant studies; (3) application of explicit inclusion and exclusion criteria; (4) appraisal of the methodological quality and the results of individual studies using clear criteria; (5) summary of the results incorporating sufficient detail for readers to make up their own minds about their interpretation; (6) recommendations or conclusions that are clearly based on the evidence presented in the review and explicit statement of values.

\section{Assessment of public health implications of sleep apnoea}

From a public health perspective, it is necessary to answer four key questions to provide patients with an equitable and effective health service: What is sleep apnoea and how common is it? Is there a causal link with morbidity and mortality? Are there cost effective and acceptable treatments available? How can we identify patients who will benefit sufficiently from treatment to justify the resources used?

WHAT IS SLEEP APNOEA AND HOW COMMON IS IT? Even with the first question we run into some confusion and uncertainty. Until recently a common definition used an apnoea/hypopnoea index (AHI) of greater than 15. However, population based studies in the USA and Australia have found that $10-20 \%$ of middle aged men have sleep apnoea according to this criterion. ${ }^{58}$ Also, there is poor correlation between AHI and symptoms of sleepiness. For example, in one epidemiological study the prevalence of excessive daytime sleepiness was found to be $41 \%$ in people with sleep disordered breathing, but this was not significantly greater than in those without sleep disordered breathing where the prevalence was $37 \%{ }^{8}$

Excessive daytime sleepiness is thus very common, as is having an AHI of more than 15 . By chance, therefore, many people will have both, even without there being a causal relationship between sleep apnoea and sleepiness. In addition, many patients with major symptoms of sleepiness caused by sleep apnoea have an AHI of less than 15. A definition which incorporates apnoeas, hypopnoeas, upper airway resistance, and symptoms of daytime sleepiness has replaced the arbitrary and narrow earlier definition. However, once sleepiness becomes integral to the definition, we cannot discuss the causal relationship between sleep apnoea and sleepiness as this is now tautological. It also creates uncertainty as to how we classify severity of disease. Furthermore, in elderly populations the prevalence of sleep disordered breathing has been found to be between $20 \%$ and $60 \%$, making it almost abnormal not to have it. ${ }^{9}$

Definitional uncertainties create great difficulty when we come to consider the possible population impact and the likely cost of an equitable health service which will meet the health needs of a population. Importantly, definitions are probably more sensibly developed in the context of the impact of treatments on length and quality of life.

IS THERE A CAUSAL LINK BETWEEN SLEEP APNOEA AND MORBIDITY AND MORTALITY?

We know from people's personal experience, and from observing the disruption to sleep that accompanies apnoeas, that sleep apnoea can cause sleepiness. This is the main reason why patients are referred for investigation and treatment. Our systematic review set about to
Correspondence to: Dr J Wright. 
investigate whether the claims being made about the effect of sleep apnoea on physical morbidity and mortality were accurate.

In assessing the evidence for causality we rely on a number of defined criteria as follows: (1) temporal relationship, (2) strength of association, (3) dose response, (4) consistency in different studies, (5) biological plausibility, and (6) cessation of exposure. These are illustrated by the relation between smoking and lung cancer. Lung cancer has been shown to follow exposure to smoking, the association is strong (measured by relative risk or odds ratio), the more a person smokes the greater their risk of developing cancer, the relationship is found consistently in different studies, biological plausibility follows from our knowledge about carcinogens in tobacco smoke, and the risk of developing lung cancer (or heart disease) declines after quitting smoking.

When considering studies which explore causal relationships, three other important issues must be considered in assessing their methodological quality-bias, chance, and confounding. These issues arise due to weaknesses in the design or conduct of research studies. Sources of bias include: (1) selection bias when the inclusion of patients depends in some way on the exposure of interest (in this case sleep apnoea); (2) surveillance bias when more careful monitoring of individuals categorised with sleep apnoea results in picking up more disease; (3) classification bias due to systematic errors in the categorisation of disease or exposure; (4) information bias which includes interviewer bias, recall bias, nonresponse bias, and bias in abstracting data.

The role of chance as an explanation for associations between conditions and outcomes can be minimised by ensuring adequate power through large enough sample sizes and can be explored by statistical analysis.

The third key problem in epidemiological studies is that of confounding, when the observed association is not causal but is mediated through a third independent factor. Important confounding factors to be considered in studies of sleep apnoea include obesity (measured by body mass index or, as a more accurate marker of central obesity, collar size or girth/height ratio), age, alcohol, and smoking.

The systematic review ${ }^{1}$ identified 54 epidemiological studies investigating the effects of sleep apnoea. Most were poorly designed and only weak or contradictory evidence was found of an association between sleep apnoea and hypertension, mortality, cardiovascular disease, pulmonary hypertension, and stroke. For example, when we assess the studies looking at the link with hypertension using the criteria for evidence of causation we find: (1) all 18 studies reviewed were cross-sectional and therefore unable to define the temporal relationshipthat is, that sleep apnoea preceded the development of hypertension; (2) the relationship was generally weak (as measured by odds ratios near 1), particularly in comparison to other risk factors such as obesity and age;(3) the dose response relationship was contradictory, with such relationship being found in four studies and not in five; (4) there was poor consistency between studies with only eight of 18 showing an association with daytime hypertension; (5) there was biological plausibilityacute spikes in blood pressure accompany apnoeas. However, nocturnal blood pressure is lower than daytime blood pressure and the relationship between these acute spikes and hypertension or vascular morbidity has not been established; (6) bias was inadequately dealt with in some studies which used selected groups and single blood pressure measurements as a marker of hypertension. Adjustment for confounding factors such as obesity, age, diet, salt intake, exercise, alcohol intake, and smoking was inadequate; (7) the two controlled intervention studies that examined the effect of CPAP failed to show any benefit after treatment (cessation) of sleep apnoea.

One consequence of the inconsistency between studies is that, in conventional reviews of the relationship between sleep apnoea and hypertension, authors can subsequently quote those studies which support their own prejudice.

Even in studies which looked at a link with road traffic accidents, the evidence, though stronger, was inconclusive. We concluded that, given the state of our knowledge, the impact of sleep apnoea on public health had been exaggerated.

ARE THERE EFFECTIVE TREATMENTS AVAILABLE? Clinicians are generally enthusiastic about their work and believe that what they are doing is best for their patients. To think otherwise would be unethical. However, this is not usually a sufficient basis for assessing the best treatment.

\section{CPAP}

CPAP is the most widely established and accepted treatment. There are some examples where the effects of a clinical condition are so great, the natural history so well known, and the effect of a treatment so large and obvious that controlled trials are not really necessary to demonstrate benefit or harm. This may be the case with very severe sleep apnoea but most patients do not fall into this category, and clinical observation alone is insufficient to provide a reliable estimate of size of benefit corresponding to different degrees of severity. It is therefore unwise for clinicians who see severe cases to generalise their observations to a wider clinical population. Good randomised controlled trials are needed to evaluate the benefits of treatment for these patients.

Our review ${ }^{1}$ assessed the 45 identified experimental studies of CPAP. Thirty nine of these were simple before and after studies; they had no control group and so it is not possible to attribute the effect to the intervention. Only one trial was a randomised controlled trial. ${ }^{10}$ Though a good trial, it was small, and we highlighted a number of methodological weaknesses. These were not nasty criticisms and, in fact, some of the weaknesses identified were likely to have led to an underestimate of effectiveness of CPAP. The main criticism was the 
lack of a comparable placebo (a pill was used). We know that the placebo effect is very powerful, and we know so little about how it works that to make presumptions about it would be unwise. For example, the presence of a wire mesh used in the treatment of incontinence has been shown to change sleep pattern $\mathrm{s}^{11}$ and placebo treatment is able to lower nocturnal blood pressure. ${ }^{12}$ Despite a large number of poorly designed studies, we concluded that the evidence strongly suggests that CPAP is effective in reducing sleepiness, probably mostly in patients with severe sleep apnoea.

Our findings were echoed in nine out of 10 summary recommendations from a recent report by the Australian Medical Research Council. ${ }^{13}$ It too recommended long term prospective studies into morbidity and mortality from sleep apnoea and randomised controlled trials to establish the benefit of CPAP to those patients with mild or moderate sleep apnoea (the majority of patients). The authors concluded, like us, that there was strong evidence for the effectiveness of CPAP in patients with severe disease. They used the results of a metaanalysis of before and after studies to recommend thresholds of the AHI above which patients should receive treatment. However, before and after studies are subject to selection bias, reporting bias, regression to the mean, temporal changes, and learning effects and, in our view, the validity of this rather unusual meta-analysis is questionable.

\section{Surgery}

A different systematic review of uvulopharyngopalatoplasty and other surgical treatments concluded that, at best, the operation was effective in less than half of patients. ${ }^{14}$ In addition, there was no valid method of identifying pharyngeal narrowing or collapse to predict which patients would benefit. There were no randomised controlled trials of this treatment and the studies that had been conducted were methodologically weak, had small sample sizes (a mean of 21), and poor validity and comparability of the outcomes used to evaluate effectiveness.

\section{Oral appliances}

As well as many before and after studies, there have been three randomised controlled trials evaluating anterior mandibular positioning devices in comparison with CPAP. These have included 72 patients, more than had been studied in trials of CPAP alone. ${ }^{15-17}$ They showed a greater reduction in AHI than with CPAP, and significant improvement in sleepiness with both treatments. It is noteworthy that there was a strong patient preference for oral appliances in these trials. However, there have not been sufficiently rigorous comparative trials to reach reliable clinical conclusions.

\section{Treating obesity}

Most patients with sleep apnoea are obese. The association between severity of disease and obesity is very strong and consistent. From our understanding of the pathophysiology and the role of fat deposits around the upper airway, this association is causal. Losing weight not only improves sleep apnoea, but can cure it. ${ }^{18}$ So, for most patients sleep apnoea is a symptom of obesity and it is common sense that, where possible, we should treat the underlying cause rather than the symptom.

This is particularly true from a public health perspective since the causal association between obesity and vascular disease is strong and well established. Not only can we reduce sleep apnoea by treating the subject's obesity, but also we can reduce the risk of heart disease and stroke. This approach could improve the health of the population as well as of the individual.

Unfortunately there are no magic pills, machines, or operations to treat obesity. Doctors tend to be nihilistic about getting patients to lose weight. It is not surprising that common advice to "go away and lose weight" rarely works. However, there are effective interventions to reduce obesity and these have been highlighted in recent reports from the NHS Centre for Reviews and Dissemination and the Royal College of Physicians. ${ }^{19}{ }^{20}$ These include cognitive therapy-including cue avoidance and behavioural therapy techniques-providing accurate nutritional and exercise advice, meal plans, weight charts, and extended contact with a therapist. However, it is unlikely that those who are usually involved in first line treatment of sleep apnoea, such as respiratory physicians, have sufficient training or experience to treat obesity effectively.

\section{What research do we need?}

Sleep apnoea, depending on the definition, is common and its impact on the health of the public is therefore potentially significant. However, there is much we need to clarify before we can justify claims for widespread investment in sleep services. We need long term prospective studies to investigate the impact of sleep apnoea on morbidity and mortality. We need well designed large randomised controlled studies to determine not only the effectiveness of CPAP and other treatments for sleep apnoea, but also to define better which patients benefit from CPAP, by how much, at what cost, and how they may be identified. We need similar high quality evaluations of other treatments.

The results of this research are likely to be more reliable if those responsible for the design and conduct are truly uncertain of the answers - that is, in genuine equipoise-so as not to introduce biases, either consciously or unconsciously.

\section{Competing priorities}

We share concerns over the way in which some health authorities have interpreted the results of our review rather simplistically. However, open debate is what is needed and this should not be censured for fear of hearing what we do not want to hear. The response of some of the enthusiasts for CPAP to our review, rather than promoting the cause of patients with sleep apnoea, creates the impression that they are not amenable to a considered approach. 
The health service in the UK has strictly limited resources and there is no shortage of competing priorities and new medical technologies. Funding effective sleep services may mean less resources to treat patients with asthma, chronic lung disease, or lung cancer. To make these decisions we need reasoned argument based on evidence of cost effectiveness and not selective opinion.

Sleep disordered breathing is a relatively new field and our understanding of it is in its infancy. If we look at other common diseases such as coronary heart disease and the effectiveness of thrombolysis, aspirin, angioplasty, and coronary artery bypass surgery we find trials of hundreds or thousands of patients. These studies tell us which patients benefit, how great this benefit is, and what the costs of treatment are-the questions that need to be answered for sleep apnoea. We should not be surprised then when recommendations for whole new services for sleep disordered breathing on the basis of trials with only 30 patients encounter some resistance.

Some of the gaps that we identified in our review are being addressed and we look forward to the results of prospective cohort studies and further randomised controlled trials of CPAP, some of which are currently underway. These will help us to make rational and informed decisions about the provision of sleep services in the UK.

1 Wright J, Johns R, Watt I, et al. Health effects of obstructive sleep apnoea and the effectiveness of continuous positive airways pressure: a systematic review of the research evidence. BMF 1997;314:851-60.

2 Phillipson EA. Sleep apnoea: a major public health problem. $N$ Engl f Med 1993;328:1271-3.

3 Douglas NJ, Polo O. Pathogenesis of obstructive sleep apnoea/hypopnea syndrome. Lancet 1994;344:653-5.
4 Simonds AK. Sleep studies of respiratory function and home respiratory support. BM7 1994;309:35-41.

5 Young T, Palta M, Dempsey J, et al. The occurrence of leep-disordered breathing among middle-aged adults. $N$ Engl f Med 1993;328:1230-5.

6 Mulrow CD. The medical review article: state of the science. Ann Intern Med 1987;106:485-8.

7 Kripke DF, Ancoli-Israel S, Klauber MR, et al. Prevalence of sleep-disordered breathing in ages 40-64 years: a population-based survey. Sleep 1997;20:65-76.

8 Olson LG, King MT, Hensley MJ, et al. A community study of snoring and sleep-disordered breathing. Prevalence. Am f Respir Crit Care Med 1995;152:711-6.

9 Ancoli-Israel S, Coy T. Are breathing disturbances in the elderly equivalent to sleep apnoea syndrome? Sleep 1994;17:77-83.

10 Engleman HM, Martin SE, Deary IJ, et al. Effect of continuous positive airway pressure treatment on daytime function in sleep apnea/hypopnoea syndrome. Lancet 1994;343:572-5.

11 Sforza E, Addati G, Cirignotta F, et al. Natural history of sleep apnoea syndrome: a five year longitudinal study. Eur Respir $\mathcal{F} 1994 ; 7: 1765-70$.

12 Mayer J, Weichler U, Cassel W, et al. Does placebo treatment lower nocturnal blood pressure in patients with sleeprelated breathing disorders and arterial hypertension? Cardiology 1993;82 (Suppl):69-78.

13 Australian Health Technology Advisory Committee. The effectiveness and cost effectiveness of nasal continuous positive airways pressure (nCPAP) in the treatment of positive airways pressure (nCPAP) in the treatment of obstructive sleep

14 Schechtman KB, Sher AE, Piccirillo JF. Methodological and statistical problems in sleep apnea research: the literature on uvulopalatopharyngoplasty. Sleep 1995;18:659-66.

15 Clark GT, Blumenfeld I, Yoffe N, et al. A crossover study comparing the efficacy of continuous positive airway pressure with anterior mandibular positioning devices on patients with obstructive sleep apnea. Chest 1996;109: 1477-83.

16 Ferguson KA, Ono T, Lowe AA, et al. A randomized crossover study of an oral appliance vs nasal-continuous positive airway pressure in the treatment of mild-moderate obstructive sleep apnea. Chest 1996;109:1269-75.

17 Ferguson KA, Ono T, Lowe AA, et al. A short term controlled trial of an adjustable oral appliance for the treatment of mild to moderate obstructive sleep apnoea. Thorax mild to moderat

18 Smith PL, Gold AR, Meyers DA, et al. Weight loss in mildly to moderately obese patients with obstructive sleep apnoea. Ann Intern Med 1985;103:850-5.

9 NHS Centre for Reviews and Dissemination. The prevention and treatment of obesity. Effective Health Care 1997;3: $1-11$.

20 Royal College of Physicians. Overweight and obese patients: principles of management with particular reference to the use of drugs. London: Royal College of Physicians, 1997. 